\title{
Predictors of Outcome After Catheter Ablation of Premature Ventricular Complexes
}

\author{
KAZIM BASER, M.D., HATICE DUYGU BAS, M.D., DIEGO BELARDI, M.D., \\ MIKI YOKOKAWA, M.D., ERIC GOOD, D.O., RAKESH LATCHAMSETTY, M.D., \\ FRED MORADY, M.D., and FRANK BOGUN, M.D.
}

From the Division of Cardiovascular Medicine, University of Michigan, Ann Arbor, Michigan, USA

Outcomes of Catheter Ablation of PVC. Background: The purpose of this study was to assess how well acute procedural outcomes predict the clinical outcome of catheter ablation of premature ventricular complexes (PVCs).

Methods: A consecutive series of 50 patients (28 women, age: $51 \pm 13$ years) with frequent PVCs was referred for PVC ablation. Acute failure was defined as inability to eliminate the predominant PVC or recurrence of the predominant PVC within 12 hours. The PVC burden was reassessed 3 months after the ablation procedure. A successful procedure was defined as reduction of the PVC burden at 3 months by $\geq 80 \%$ of the initial burden.

Results: The procedure was acutely effective in 37 patients (74\%) and at 3 months in 40 patients $(80 \%)$. The presence or absence of the predominant PVC in the 12 hours postablation had the highest accuracy for outcome at 3 months (accuracy: 90\%). From among the 13/50 patients (26\%) with evidence of acute failure, 4 had a PVC reduction of $\geq 80 \%$ at 3 months and 10 had a PVC reduction of $>50 \%$ resulting in symptomatic improvement at 3 months.

Conclusion: The presence or absence of the predominant PVC within 12 hours postablation best correlated with the 3-month-efficacy data. Recurrence of the predominant PVC shortly after ablation did not indicate a procedural failure and the necessity for a repeat procedure. The majority of these patients had a significant, clinically meaningful reduction in their PVC burden. Acute predictors for procedural outcome at 3 months have a high positive but rather low negative predictive value. ( $\mathrm{J}$ Cardiovasc Electrophysiol, Vol. 25, pp. 597-601, June 2014)

premature ventricular complexes, catheter ablation, outcome

\section{Background}

Frequent premature ventricular complexes (PVCs) have been targeted by radiofrequency ablation in patients with and without structural heart disease..$^{1-3}$ The factors that correlate with PVC recurrence have not been systematically described. It is unclear whether periprocedural elimination of a predominant PVC is required for a successful outcome. The purpose of this study was to assess determinants of 3-month efficacy in patients who underwent ablation of frequent PVCs.

\section{Methods}

\section{Patient Characteristics}

In a consecutive series of 50 patients, (28 women, age: $51 \pm 13$ years, ejection fraction: $52 \pm 12.6 \%$; Table 1) with frequent idiopathic PVCs who were referred for ablation, telemetry was performed postablation for 12 hours.

Dr. F. Bogun has received a grant from the Leducq foundation.

No disclosures.

Address for correspondence: Frank Bogun, M.D., Cardiovascular Center, SPC 58531500 E. Medical Center Drive, Ann Arbor, MI 48109-5853, USA. Fax: 734-936-7026; E-mail: fbogun@med.umich.edu

Manuscript received 4 November 2013; Revised manuscript received 8 February 2014; Accepted for publication 10 February 2014.

doi: $10.1111 /$ jce. 12400
Patients with structural heart disease were excluded. Patients failed $1 \pm 1$ antiarrhythmic medications including $\beta$-blocker and calcium channel blocker therapy, and $31 / 50$ patients $(62 \%)$ had undergone a prior failed attempted ablation procedure. Forty-two patients had palpitations and the remaining 8 patients had an impaired left ventricular ejection fraction $(38.9 \pm 9.4 \%)$, and 20 patients $(40 \%)$ had pleomorphic PVCs and the remainder had unimorphic PVCs.

\section{Holter Recordings}

A 24-hour 12-lead Holter was recorded before the ablation procedure to determine the PVC burden. The recording was repeated 3 months postablation. A predominant PVC morphology was defined as a PVC accounting for $>80 \%$ of all PVC morphologies based on a 12-lead Holter recording. A predominant PVC was present in $46 / 50$ patients; in the remaining 4 patients the predominant PVC accounted for $75 \pm$ $2 \%$ of all PVC morphologies. Immediately postprocedure, the patients were connected to a 7-lead-telemetry unit that was stored (General Electrics, CIC, Milwaukee, WI, USA) and all patients were observed for at least 12 hours after ablation. Each PVC was subsequently analyzed as to whether it was the targeted PVC morphology or another PVC morphology. The PVCs were quantified for 12 hours postprocedure and the postprocedural PVC burden was compared to the preprocedural PVC burden. Before ablation, variability of the PVC burden was assessed with 2 different Holters $70 \pm$ 121 days apart. The PVC burden of both Holters was similar $(\mathrm{P}=0.48)$. 


\section{TABLE 1}

Patient Characteristics

\section{Variable}

Patients, $\mathrm{n}$

Age (years)

Male gender, $\mathrm{n}$

Left ventricular ejection fraction (\%)

PVC burden (\%)

Therapy, $\mathrm{n}$

$\beta$-Blockers

Calcium channel blockers

Antiarrhythmic drug therapy including amiodarone

Data are shown as mean \pm 1 standard deviation. Percentage values are shown in parentheses. $\mathrm{PVC}=$ premature ventricular complex.

\section{Electrophysiology Study}

After informed consent was obtained, 3 quadripolar electrode catheters were advanced via a femoral vein to the right ventricular apex, the His position, and the high right atrium. After vascular access was obtained, 3,000 units of heparin was administered. If arterial access was also obtained, 5,000 units of heparin was given targeting an activated clotting time of 250-300 seconds. Programmed ventricular stimulation with up to 4 extrastimuli was performed to induce ventricular tachycardia. Surface electrocardiographic leads were recorded in combination with bipolar intracardiac tracings and recorded on optical disc (EP Med Systems, West Berlin, NJ, USA). Amount and the type of medication used during the ablation procedure (midazolam, propofol, ketamine, morphine, remifentanyl, or fentanyl) were recorded and compared among the groups with failed and effective procedures. Only conscious sedation and no general anesthesia was used during the ablation procedures.

\section{Mapping and Ablation}

A 3-dimensional mapping system (CARTO, Biosense Webster, Diamond Bar, CA, USA) was used for mapping in all patients. Mapping was performed with 3.5-mm-openirrigated-tip catheter (Thermocool Navistar, Biosense Webster). The site of origin was identified by activation mapping in the presence of frequent PVCs; in the absence of frequent PVCs, pace mapping was used to identify the site of origin. A site of origin was defined as intramural when pacing at the site of earliest activation from either side of the ventricular cavity failed to show a matching pace map ( $<10 / 12$ leads). ${ }^{4}$ Radiofrequency energy was delivered for $60-120$ seconds at the site of origin. An impedance drop of $10 \Omega$ was targeted with a power of 30-50 W. If radiofrequency energy was delivered in an aortic cusp or within the coronary venous system, an initial power of 15-20 W was used. The ablation endpoint was to eliminate the predominant PVCs. If other PVCs were present, they were also targeted at the discretion of the operator.

\section{Definitions}

The procedure was defined as acutely effective if the predominant PVC was eliminated and did not recur for 12 hours postablation. Recurrence of the predominant PVC within 12 hours postablation was defined as acute failure of the ablation procedure. At 3 months, a decrease by $\geq 80 \%$ of the initial
PVC burden was defined as an effective ablation procedure. A partially effective procedure was defined arbitrarily as a reduction in the PVC burden by $50-80 \%$ of the initial PVC burden. The 12-lead morphology of the PVC-QRS complex was compared to other PVC morphologies. PVCs were defined as pleomorphic if they differed in at least 3/12 ECG leads from another PVC morphology in a given patient.

\section{Follow-Up}

All patients had a Holter at 3 months postablation. They were seen in follow-up 3-6 months postablation. Subsequently patients were seen on an as-needed basis. Antiarrhythmic drugs were discontinued postablation if the procedure was successful. $\beta$-Blockers and calcium channel blockers were continued only if there was an indication other than the presence of PVCs. No new antiarrhythmic drugs were added postablation.

\section{Statistical Analysis}

Continuous variables were expressed as mean \pm 1 standard deviation and were compared with Student's $t$-test. Categorical variables were compared with the $\chi^{2}$ test. If the sample size was smaller than 5 in a given cell, Fisher's exact test was used. A P value of $<0.05$ was considered statistically significant. Paired sample $t$-tests were used to compare PVC burden before and after ablation and for PVC variability.

\section{Results}

\section{PVC Elimination and Outcome}

Elimination of the predominant PVCs during the ablation was achieved in $47 / 50$ patients $(94 \%)$. During the immediate 12-hour postprocedure, the predominant PVC did not recur in $37 / 50$ patients $(74 \%)$. At 3 months, the predominant PVC did recur in only $1 / 37$ patients with an acutely effective procedure. The PVC burden was reduced from $19 \pm 13 \%$ to $1.1 \pm$ $3.8 \%$ in the 12-hour postablation period and increased to $2.1 \pm 4.5 \%$ at 3 months postablation ( $\mathrm{P}$ values $<0.0001$ for each comparison with the initial PVC burden). Regardless of the PVC burden within 12 hours postablation, the presence or absence of the predominant PVC within 12 hours postablation had the highest accuracy $(90 \%)$ in predicting failure or complete success of the procedure at 3 months (positive predictive value: $97 \%$, negative predictive value: $70 \%$, sensitivity: 90\%, specificity: 90\%). Reduction of the PVC burden within 12 hours postprocedure (by $\geq 80 \%$ of the initial burden) predicted the 3-month efficacy with an accuracy of $82 \%$ (positive predictive value: $84 \%$, negative predictive value: 60\%, sensitivity: $95 \%$, specificity: $30 \%$ ).

In patients with an acutely successful procedure, the PVC burden 3 months postablation was significantly lower compared to patients with a failed acute procedure $(1.1 \pm 3.3 \%$ vs. $5.1 \pm 6.4 \%, \mathrm{P}=0.0001$; Fig. 1$)$.

At 3 months, $40 / 50$ patients $(80 \%)$ had a reduction of $\geq 80 \%$ of the initial PVC burden and in a total of $46 / 50$ patients $(92 \%)$ the procedure reduced the initial PVC burden by at least $50 \%$.

With respect to procedural complications, 3 patients had groin hematomas (no surgery required), 1 patient had pericardial tamponade necessitating pericardiocentesis, and 


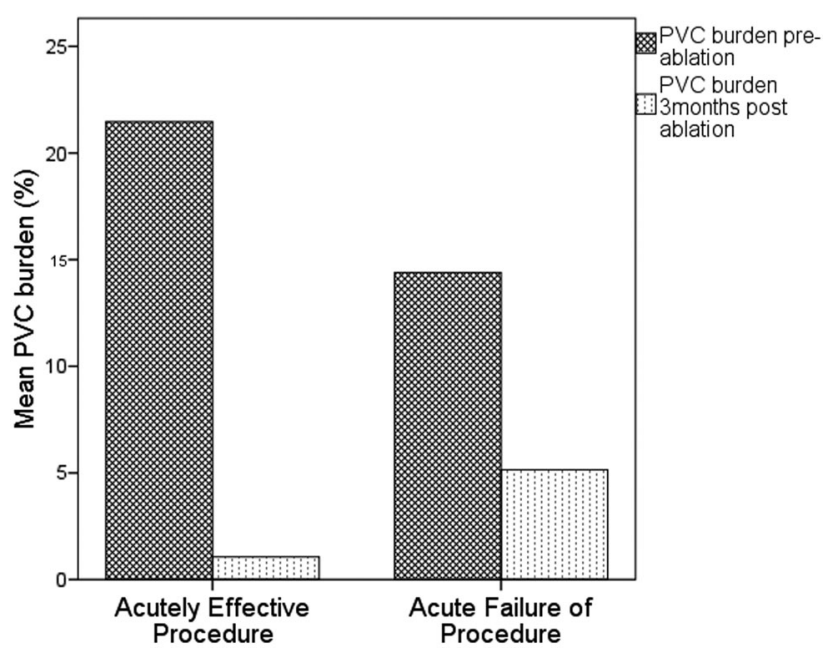

Figure 1. Preablation and 3 months postablation PVC burden in patients with an acutely effective versus an acutely failed procedure. There was no significant difference in the PVC burden preablation. The PVC burden at 3 months postablation was significantly reduced.

1 patient had a coronary venous dissection with minimal pericardial effusion that was managed conservatively.

\section{Failed Acute Ablation}

The procedure acutely failed in $13 / 50$ patients (26\%). In $3 / 13$ patients, the predominant PVCs could not be eliminated during the ablation. A total 2 of these 3 patients had failed procedures at 3 months. In 1/3 patients, PVCs disappeared 9 hours postablation and at the 3-month follow-up Holter there was a $\geq 80 \%$ reduction compared to the initial PVC burden. The PVC burden before the ablation in these 3 patients changed from $15 \pm 11 \%$ preprocedure to $9 \pm 12 \%$ at 3 months (ns).

On the other hand, in 10 patients $(20 \%)$ the predominant PVC was eliminated during the ablation procedure and did not recur within 1 hour postablation, yet PVCs returned within 12 hours postprocedure. However, in $3 / 10$ patients the PVC burden was reduced by $\geq 80 \%$ of the initial PVC burden at 3 months and in $8 / 10$ patients the PVC burden was reduced by $>50 \%$ of the initial PVC burden preablation at 3 months.

Thus in 10/13 patients (77\%), the PVC burden at 3 months was reduced by at least $50 \%$ and by $\geq 80 \%$ of the initial burden in $4(29 \%)$ patients with acutely failed procedures. The PVC burden in these 13 patients was reduced from $14.4 \pm$ $11.0 \%$ to $5.1 \pm 6.4 \%(\mathrm{P}<0.05)$.

The patients with effective or partially effective ablation reported improvement of symptoms postablation.

There was no significant difference in whether or not sedation was administered during the ablation procedure or which sedative was used (midazolam vs. propofol vs. fentanyl) when patients with and without acute failure were compared.

\section{Pleomorphic PVCs and Predominant PVCs}

Less than half of the patients (20 patients; $40 \%$ ) had pleomorphic PVCs at the time of the ablation procedure. The mean number of PVC morphologies per patient was $2.8 \pm$ 3.3 (range: $1-15$ ) and $1.34 \pm 0.7$ PVC morphologies were mapped per patient (range: 1-4 per patient). A predominant PVC, however, was present in the preprocedural Holter in $46 / 50$ patients. The predominant PVC accounted for a mean of $96 \%$ of the entire PVC burden (range: 73-100\%). Despite targeting the predominant PVC in all patients, in patients with pleomorphic PVCs the success rate was lower (65\% vs. $90 \%, \mathrm{P}=0.036$ ) at 3 months. There was, however, no difference when patients with and without pleomorphic PVCs were compared with respect to acute outcome $(65 \%$ vs. $80 \%, \mathrm{P}=$ 0.33 ). A nonpredominant PVC became the predominant PVC in 1/20 patients with pleomorphic PVCs during follow-up.

\section{PVC Origin and Outcome}

The site of origin of the predominant PVC (Fig. 2, Table 2) was from the right ventricular outflow tract $(n=$ $11)$, an intramural focus $(n=9)$, the epicardium $(n=7)$, the papillary muscles $(n=6)$, the pulmonary artery $(n=$ $7)$, the aortic cusps $(n=2)$, the left ventricular outflow tract $(n=3)$, the fascicles $(n=2)$, the mitral annulus $(n=1)$, the aorto-mitral continuity $(n=1)$, and the tricuspid annulus

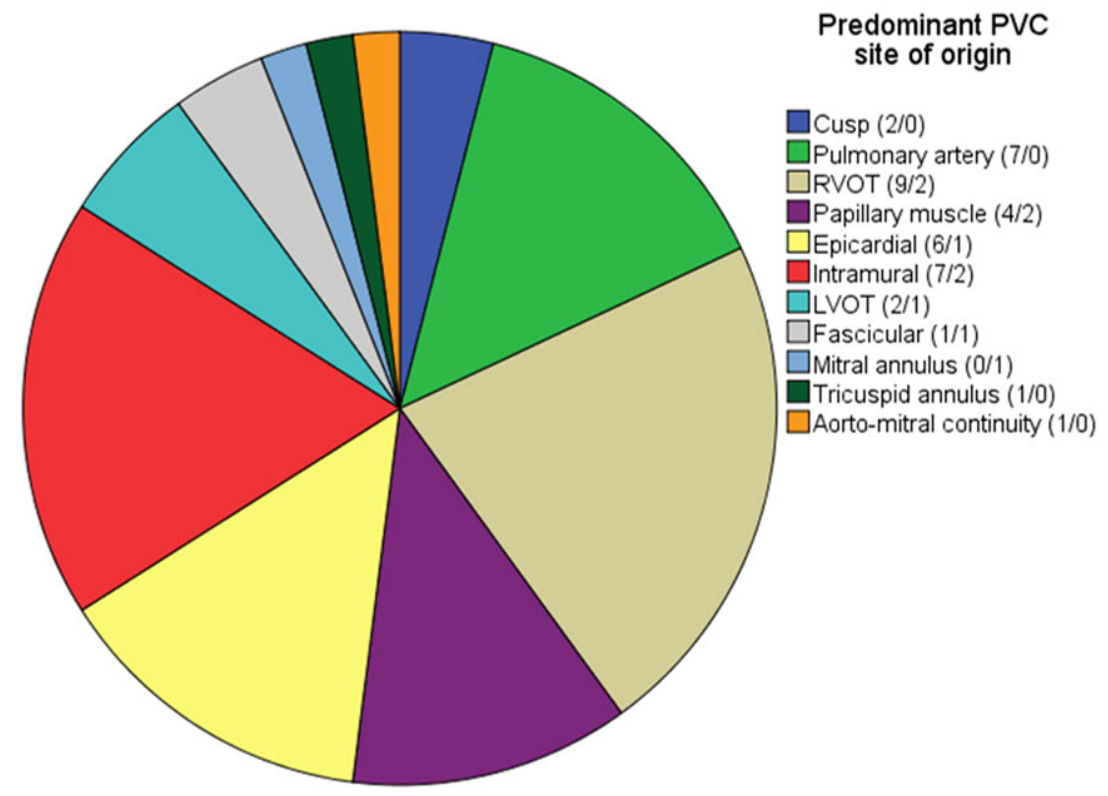

Figure 2. Site of origin of predominant PVCs. The numbers in parentheses indicate number of patients with success/failure at 3 months postablation. For a high quality, full color version of this figure, please see Journal of Cardiovascular Electrophysiology's website: www.wileyonlinelibrary.com/journal/jce 
TABLE 2

Acute and 3-Month Success Rates with Respect to the Site of Origin of PVCs

\begin{tabular}{lcccc}
\hline & $\begin{array}{c}\text { Acutely } \\
\text { Effective } \\
\text { Procedure }\end{array}$ & $\begin{array}{c}\text { Acute } \\
\text { Failure }\end{array}$ & $\begin{array}{c}\text { 3 Month } \\
\text { Success }\end{array}$ & $\begin{array}{c}\text { 3 Month } \\
\text { Failure }\end{array}$ \\
\hline Cusp, 2 & $2(100)$ & 0 & $2(100)$ & 0 \\
Pulmonary artery, 7 & $6(86)$ & $1(14)$ & $7(100)$ & 0 \\
RVOT, 11 & $9(82)$ & $2(18)$ & $9(82)$ & $2(18)$ \\
Papillary muscle, 6 & $3(50)$ & $3(50)$ & $4(67)$ & $2(33)$ \\
Epicardial, 7 & $6(86)$ & $1(14)$ & $6(86)$ & $1(14)$ \\
Intramural, 9 & $7(78)$ & $2(22)$ & $7(78)$ & $2(22)$ \\
LVOT, 3 & $2(67)$ & $1(33)$ & $2(67)$ & $1(33)$ \\
Fascicular, 2 & 0 & $2(100)$ & $1(50)$ & $1(50)$ \\
Mitral annulus, 1 & 0 & $1(100)$ & 0 & $1(100)$ \\
Tricuspid annulus, 1 & $1(100)$ & 0 & $1(100)$ & 0 \\
Aorto-mitral continuity, 1 & $1(100)$ & 0 & $1(100)$ & 0 \\
Total, 50 & $37(74)$ & $13(26)$ & $40(80)$ & $10(20)$ \\
\hline Percnin, &
\end{tabular}

Percentage values are shown in parentheses. The indicated numbers of success and failure at 3 months do not include the numbers of acute results. LVOT $=$ left ventricular outflow tract; $\mathrm{PVC}=$ premature ventricular complex; $\mathrm{RVOT}=$ right ventricular outflow tract.

$(n=1)$. In 2 patients the site of origin of the predominant PVC was the aortic cusps, in 3 the left ventricular outflow tract, fascicular in 2 patients, the mitral annulus in 1 patient, aortomitral continuity in 1 patient, and tricuspid annulus in 1 patient. In 5 patients, there was a late improvement in the PVC burden at 3 months follow up. The site of origin of the PVCs in these patients were: the papillary muscles $(n=2)$, the pulmonary artery $(n=1)$, the epicardium $(n=1)$, and the fascicular system $(n=1)$. No site of origin was predictive of the outcome at 3 months postablation $(\mathrm{P}=0.27)$.

\section{Discussion}

In this study of patients with frequent PVCs, targeting the predominant PVC morphology resulted in a substantial reduction of the PVC burden in almost $90 \%$ of patients. The strongest predictor of 3-month efficacy was the absence of the predominant PVC during the 12-hour period postablation. However, persistence or recurrence of the predominant PVC early postablation did not predict procedural failure at 3 months.

\section{Predictors of Outcome: Acute Effective Ablation}

Elimination of the targeted PVC for 12 hours postprocedure was the best predictor for a favorable 3-month outcome. In the patients with acute success, the site of origin of the PVC was reached and eliminated. Recurrence was rare if the PVCs did not recur within the periprocedural observation period and was documented in only 1 patient with acute procedural success. It is unlikely that day-to-day variations of the PVC burden accounted for the decrease in the PVC burden acutely and at 3 months postablation since the variation of the PVC burden before the ablation was not significant based on 2 preprocedural Holters. Furthermore, the stability of the PVC burden in patients without ablation has been reported over a much longer time period than in this study. ${ }^{5}$ Also, type and amount of sedation given during the ablation procedure did not differ between patients, and sedation is therefore unlikely to have resulted in the acute change of the PVC burden postprocedure.

\section{Predictors of Outcome: Acute Failed Ablation}

Complete elimination of the predominant PVC for 12 hours postablation or for even 1 hour postablation may not be possible in every patient; and patients with frequent PVCs may still benefit from an ablation and have symptomatic improvement, even if the PVC burden is only reduced by $50 \%$ rather than $80 \%$ of the initial burden. In about $70 \%$ of the patients in whom acute outcome measures suggested a procedural failure, the PVC burden was substantially reduced at the 3-month follow-up. It appears that patients in whom elimination of the PVCs during the procedure was achieved despite their recurrence within 12 hours had a better outcome than the patients in whom this was not possible.

Procedural failure can be divided into the inability to completely eliminate the predominant PVC during the ablation procedure and recurrence of the predominant PVC within 12 hours postprocedure. In the former group of patients, the site of origin was not reached or originated close to the conduction system. In these patients, elimination of PVCs was not seen acutely and patients still had a high PVC burden at the 3-month follow-up Holter.

In the latter form where PVCs recurred after elimination of the PVCs during the ablation procedure, edema formation during radiofrequency energy delivery might have accounted for the disappearance of the targeted PVC acutely and its recurrence later in the early postablation period. The arrhythmogenic substrate was altered, since the 3-month follow-up showed a significant reduction of the preprocedural PVC burden in the patients with early recurrence of PVCs. Lesion maturation may be a factor that accounts for this observation. ${ }^{6}$

\section{Predominant PVC and Other PVCs}

For ventricular tachycardia (VT), complete elimination is used as a definition for acute success and a single recurrence is defined as failure. For PVCs, a less stringent definition of reduction of the PVC burden by at least $80 \%$ of the initial burden is often used accounting for pleomorphic PVCs and the fact that most often a predominant PVC is targeted only. In the majority of the patients in this series there was indeed a predominant PVC, and the strategy to eliminate the predominant PVC was effective in most patients and resulted in clinical benefit in almost $90 \%$ of patients. However, pleomorphic PVCs remain a problem and, not unexpectedly, the success rate in these patients is lower. It remains to be determined whether targeting nonpredominant PVCs will increase the success rate at 3 months. Dynamic changes in the immediate postprocedural period may account for the rise in the PVC burden from 12 hours postablation to 3 months postablation. And therefore it is not surprising that the reduction of the PVC burden at 12 hours postablation was the least accurate predictor of the 3-month outcome.

Assessment of the procedural success may not be possible right after the procedure and may take up to 3 months especially if the predominant PVC recurs postablation. Since patients had clinical benefit even if the PVC burden was not reduced by $\geq 80 \%$ of the initial value, we propose to also 
use a definition of partial success where the PVC burden has been reduced to a lower level.

\section{Limitations}

A 7-lead recording system was used for postprocedure telemetry. It is therefore possible that the PVC morphologies might not have been accurately classified as the predominant or nonpredominant PVC, especially in patients with pleomorphic PVCs. However, a PVC was only defined as the predominant PVC on telemetery if it had the same bundle branch block morphology, the same axis and other similar morphology characteristics compared to the 12-lead ECG morphology of the predominant PVC. Since repeat Holters were not performed for all patients postablation, it is possible that a normal variation might have accounted for a reduced PVC burden in some individual patients. This is a small series of patients including patients with previously failed ablations and patients with PVCs originating from papillary muscles as well as the epicardium. A longer follow-up period is required especially in patients with pleomorphic PVCs to determine the value of targeting only the predominant PVC.

\section{Conclusions}

Elimination of the predominant PVC for a period of 12 hours postprocedure best predicts the 3-month outcome for PVC ablation. Recurrence of the predominant PVC shortly after ablation does not indicate a procedural failure and the necessity for a repeat procedure. Yet the majority of these pa- tients still had a significant, clinically meaningful reduction in their PVC burden. Acute outcomes measures are imperfect in predicting 3-month outcomes post-PVC ablation.

\section{References}

1. Bogun F, Crawford T, Reich S, Koelling TM, Armstrong W, Good E, Jongnarangsin K, Marine JE, Chugh A, Pelosi F, Oral H, Morady F: Radiofrequency ablation of frequent, idiopathic premature ventricular complexes: Comparison with a control group without intervention. Heart Rhythm 2007;4:863-867.

2. Sarrazin JF, Good E, Kuhne M, Oral H, Pelosi F Jr, Chugh A, Jongnarangsin K, Crawford T, Ebinger M, Morady F, Bogun F: Mapping and ablation of frequent post-infarction premature ventricular complexes. J Cardiovasc Electrophysiol 2010;21:1002-1008.

3. Mountantonakis SE, Frankel DS, Gerstenfeld EP, Dixit S, Lin D, Hutchinson MD, Riley M, Bala R, Cooper J, Callans D, Garcia F, Zado ES, Marchlinski FE: Reversal of outflow tract ventricular premature depolarization-induced cardiomyopathy with ablation: Effect of residual arrhythmia burden and preexisting cardiomyopathy on outcome. Heart Rhythm 2011;8:1608-1614.

4. Yokokawa M, Good E, Crawford T, Chugh A, Pelosi F Jr, Latchamsetty R, Jongnarangsin K, Ghanbari H, Oral H, Morady F, Bogun F: Reasons for failed ablation for idiopathic right ventricular outflow tract-like ventricular arrhythmias. Heart Rhythm 2013;10:1101-1108.

5. Niwano S, Sasaki T, Kurokawa S, Kiryu M, Fukaya H, Hatakeyama Y, Niwano H, Fujiki A, Izumi T: Predicting the efficacy of antiarrhythmic agents for interrupting persistent atrial fibrillation according to spectral analysis of the fibrillation waves on the surface ECG. Circ J 2009;73:1210-1218.

6. Langberg JJ, Borganelli SM, Kalbfleisch SJ, Strickberger SA, Calkins H, Morady F: Delayed effects of radiofrequency energy on accessory atrioventricular connections. Pacing Clin Electrophysiol 1993;16 (Pt 1):1001-1005. 